International Radiation Symposium (IRS2012)

Berlin, Germany; 6-10 August, 2012.

\title{
Geophysical information from advanced sounder infrared spectral radiance
}

\author{
Allen M. Larar ${ }^{1}$, Daniel K. Zhou ${ }^{1}$, Xu Liu ${ }^{1}$, William L. Smith ${ }^{2,3}$ \\ ${ }^{1}$ NASA Langley Research Center, Hampton, VA, USA \\ ${ }^{2}$ Hampton University, Hampton, VA, USA \\ ${ }^{3}$ University of Wisconsin-Madison, Madison, WI, USA
}

\begin{abstract}
Advanced satellite sensors are tasked with improving global observations of the Earth's atmosphere, clouds, and surface to enable enhancements in weather prediction, climate monitoring capability, and environmental change detection. Satisfying this type of improvement for inferred geophysical information from these observations requires optimal usage of data from current systems as well as enhancements to future sensors. This presentation addresses the information content present in infrared spectral radiance from advanced atmospheric sounders with an emphasis on knowledge of thermodynamic state and trace species. Results of trade-off studies conducted to evaluate the impact of spectral resolution, spectral coverage, instrument noise, and a priori knowledge on remote sensing system information content will be discussed. A focus is placed on information achievable from the Atmospheric InfraRed Sounder (AIRS) on the NASA EOS Aqua satellite in orbit since 2002, the Infrared Atmospheric Sounding Interferometer (IASI) aboard MetOp-A since 2006, and the Cross-track Infrared Sounder (CrIS) instrument aboard the NPP and JPSS series of satellites which began 28 October 2011.
\end{abstract}

